\title{
The association of a simple blood pressure- independent parameter derived from ambulatory blood pressure variability with short-term mortality
}

\author{
Benjamin Gavish ${ }^{1,5}$, Iddo Z Ben-Dov ${ }^{2,5}$, Jeremy D Kark ${ }^{3}$, Judith Mekler ${ }^{4}$ and Michael Bursztyn ${ }^{4}$
}

We explored the predictive ability of the blood pressure variability ratio (BPVR), defined as the ratio of 24-h ambulatory systolic blood pressure variability to diastolic variability, and evaluated its predictable relation with blood pressure and the Ambulatory Arterial Stiffness Index (AASI). A total of 3433 consecutive patients were followed up to 16 years for all-cause mortality. Blood pressure variability was expressed by the standard deviation. BPVR, which is the systolic-on-diastolic slope estimated by a known type of symmetric regression ('reduced major axis'), was compared with other expressions of this slope and with AASI using other regression procedures. Time-dependent Cox proportional hazard models, adjusted for demographics, 24-h mean blood pressure, 24-h pulse pressure and dipping were used to assess the association of BPVR and slope-related parameters with all-cause mortality. We found that Pearson's correlation between BPVR and the symmetric slope was 0.957, and between 1-1/BPVR (an AASI-equivalent expression) and the symmetric version of AASI was 0.973 . BPVR was entirely independent of mean arterial pressure $(r=0.013)$. Systolic and diastolic ambulatory blood pressure variability was not significantly associated with mortality. Over 16 years, BPVR predicted all-cause mortality [hazard ratio $=1.21(95 \% \mathrm{Cl} 1.05-1.40)$ per 1 s.d. increase]. In time-dependent models, increased BPVR was strongly associated with an 18-month mortality, weakly related to 7 years mortality, showing no effect thereafter. Thus, the ratio between 24-h systolic and diastolic blood pressure variability, readily available from ambulatory monitoring reports, is an easy-to-calculate systolic-on-diastolic slope. It is a blood pressureindependent measure believed to express an arterial property, with prognostic power similar to that of AASI. Hypertension Research (2009) 32, 488-495; doi:10.1038/hr.2009.39; published online 24 April 2009

Keywords: ambulatory arterial stiffness index; blood pressure variability; mortality; prognosis

\section{INTRODUCTION}

Ambulatory blood-pressure monitoring (ABPM) provides numerous measures beyond the arithmetic mean of the measurements determined. ${ }^{1}$ Measures derived from the linear relationship between systolic and diastolic pressures are becoming the target of research efforts and are believed to be associated with mechanical properties of the arteries (a physiological model has been proposed ${ }^{2}$ ). These include systolic-on-diastolic slope (Slope) ${ }^{3}$ and 1 -(diastolic-on-systolic slope) called Ambulatory Arterial Stiffness Index (AASI). ${ }^{4}$ Slope and AASI have been shown to be independent of mean blood pressure, ${ }^{3}$ and to incorporate clinical and prognostic information. ${ }^{5-8}$

One problem in using these parameters in clinical practice is their sensitivity to the estimation method applied: originally, AASI was derived by standard regression [hence, we identify it as AASI(standard) ]. ${ }^{4}$ Standard regression is known to be an inappropriate estimation method in this case (as systolic and diastolic cannot be described as 'independent' and 'dependent' variables), ${ }^{9,10}$ leading to artifactual dependence of AASI(standard) on data scattering, and bias in relating this parameter to clinical variables. ${ }^{3}$ This bias is eliminated on deriving the regression parameters with a procedure ('bisector regression') that treats systolic and diastolic BP in a symmetrical manner. ${ }^{3}$ However, the specific method applied involves a mathematical expression that is too complex to have a simple clinical interpretation.

The rationale for this work stemmed from the finding that there is a symmetrical regression type called 'reduced major axis regression' that expresses Slope simply as the ratio between the standard deviation of the data along the $Y$ axis (systolic BP) over that of the $X$ axis (diastolic BP). ${ }^{9}, 10$ We named this ratio 'blood pressure variability ratio' (BPVR). Accordingly, by definition, AASI(BPVR) $=1-1 /$ BPVR. In this way, both parameters have a simple relationship with variability measures and their calculation does not require any statistical software! Furthermore, BP variability is a clinically meaningful parameter by itself. Ambulatory blood pressure variability, expressed by the standard deviation from the mean, is linked with mean BP levels, ${ }^{11}$ but

${ }^{1}$ InterCure Ltd, Lod, Israel; ${ }^{2}$ Nephrology and Hypertension, Hadassah-Hebrew University Medical Center, Jerusalem, Israel; ${ }^{3}$ Epidemiology Unit, Hadassah-Hebrew University Medical Center, Jerusalem, Israel and ${ }^{4}$ Internal Medicine, Mt-Scopus Campus, Hadassah-Hebrew University Medical Center, Jerusalem, Israel

${ }^{5}$ These authors contributed equally to this work.

Correspondence: Dr IZ Ben-Dov, Nephrology and Hypertension Services, Hadassah-Hebrew University Medical Center, Ein-Kerem, PO Box 12000, Jerusalem, 91120 Israel. E-mail: iddobe@ekmd.huji.ac.il

Parts of this work were presented in the ESH-ISH meeting, Berlin 2008 (poster), and the CHBPR meeting, Atlanta 2008 (poster p140).

Received 5 November 2008; revised 3 March 2009; accepted 9 March 2009; published online 24 April 2009 
independently associated with target organ damage ${ }^{12-14}$ and outcome. ${ }^{15-17}$

In this study, we used data from a clinical ABPM service to investigate the relationships between both blood pressure variability and BPVR and clinical, demographic as well as mortality outcome variables, and especially the contribution of BPVR as a predictor of all-cause mortality independently of established ABPM predictors. In addition, we evaluated the theoretical and phenomenological relationship between BPVR and Slope and AASI calculated by different regression methods.

\section{METHODS}

\section{Study population}

Data were extracted from our entire ABPM service database, from 1991 through to 2005, which included 3433 eligible patients for whom blood pressure variability and ambulatory arterial stiffness indices could be extracted from raw monitoring data files. Excluded were patients less than 16 years old, pregnant women and subjects with poor-quality ABPM recordings (less than 15 valid measurements). Individuals with repeated monitoring(s) were included only once in the analyses, using data from the initial monitoring session. Patients had been referred for standard clinical indications at the discretion of the referring physician (mainly primary-care practitioners, who have been shown elsewhere to use ABPM for appropriate indications ${ }^{18}$ ). Baseline data collected included demographic characteristics, height and weight, and treatment for hypertension and diabetes. The outcome assessed was all-cause mortality, which was obtained by linkage (dated 20 January 2008) with the national population register by way of the individual national ID number.

\section{Ambulatory blood-pressure monitoring and definitions}

Twenty-four hour ABPM was executed with Spacelabs 90207 (Redmond, WA, USA), as described earlier. ${ }^{19}$ Before 1999, we used Accutracker II (Suntech, Raleigh, NC, USA). ${ }^{20,21}$ The monitor was mounted on the non-dominant arm between 0800 and 1000 hours, and removed 24-h later. Recordings were made every $20 \mathrm{~min}$ between 0600 hours and midnight, and every $30 \mathrm{~min}$ between midnight and 0600 hours. A mercury sphygmomanometer was initially attached to the monitor through a Y-connector to verify agreement between the two modes of measurement (within a range of $5 \mathrm{~mm} \mathrm{Hg}$ ). Cuff size was selected according to measured arm circumference; up to $24 \mathrm{~cm}$ pediatric cuff, $24-32 \mathrm{~cm}$ standard adult cuff and over $32 \mathrm{~cm}$ large adult cuff. The average of 2-3 initial sphygmomanometer measurements taken by a trained technician, after the subject had been seated for $5 \mathrm{~min}$, was considered the patient's clinic $\mathrm{BP}^{22,23}$ Sleep, including daytime naps, was logged in a diary. Sleep-related BP dipping refers to the relative reduction in BP from wakefulness levels to sleep levels, according to the logs.

\section{Blood pressure variability and slope-related parameters}

Systolic and diastolic BP variability was expressed, respectively, by withinsubject standard deviation (s.d.) of systolic BP (SBP) and diastolic BP (DBP), that is, s.d.(SBP) and s.d.(DBP), as routinely included in every ABPM report. Variability was also expressed by the coefficient of variation (CV) of systolic or diastolic measurements, defined using relative changes $(\mathrm{CV}=100 \times$ s.d./mean). The BP variability ratio (BPVR) was defined as (systolic BP variability)/ (diastolic BP variability), namely, s.d.(SBP)/s.d.(DBP).

The original AASI, AASI(standard), is defined as $1-$ (diastolic-on-systolic slope), wherein the slope was determined from a DBP $v s$. SBP plot by a standard regression procedure. ${ }^{4}$ Viewed in the reciprocal SBP $v s$. DBP plot, this regression line has the slope of $1 /$ (diastolic-on-systolic slope), which is generally different from the systolic-on-diastolic slope ${ }^{10}$ (as shown in Figure A1, see Appendix). As a result, AASI(standard) displays an artifactual dependence on data scattering, as expressed by the correlation coefficient $R$ between SBP and DBP. $^{3,24,25}$ The use of symmetric regression of the bisector type (see Figure A1) ${ }^{10}$ eliminates this problem, ${ }^{3}$ with the estimated systolic-on-diastolic slope, Slope(bisect) and AASI(bisect) $=1-1 /$ Slope(bisect). The current work applies the symmetric regression of the 'reduced axis type', according to which the estimated systolic-on-diastolic slope is given by BPVR (see Figure A1), ${ }^{9,10}$ which is far simpler than the mathematical expression for Slope(bisect). Accordingly, AASI(BPVR) is given by $1-1 / \mathrm{BPVR}$. It is important to mention that the relationship, $\mathrm{AASI}$ (standard) $=1-R / \mathrm{BPVR}$, is a mathematical identity that does not require experimental validation. All relevant statistical background, including the relationship between the various parameters, is outlined in Appendix.

\section{Statistical analysis}

The distribution of systolic BP variability, diastolic BP variability and BPVR was skewed (skewness $0.911 \pm 0.042,0.885 \pm 0.042$ and $1.195 \pm 0.042$ ). Thus, log-transformed values were used to characterize relationships with other variables. Determinants of the BP variability indices were assessed by multivariable regression models including, demographic characteristics (age, sex and $\mathrm{BMI}$ ), treatment (medical treatment for hypertension and diabetes) and monitoring measures (mean arterial pressure (MAP) and MAP dip).

Analysis of outcome consisted of Cox proportional hazards models predicting all-cause mortality. However, for the variables under investigation (BPVR and slope-related parameters), the proportional hazards assumption (as assessed by including an appropriate time-dependent covariate) was not valid. Specifically, the hazards associated with increased AASI(standard), AASI(bisect) or BPVR seemed to decrease with time. Thus, Cox proportional hazards modeling with two time-dependent covariates was used. The first timedependent covariate consisted of the index measure (BPVR, AASI, and so on) multiplied by 1 for time $\geqslant 1.5$ years or by 0 for time $<1.5$ years, whereas the second covariate was for 7 years (defined similarly). Mortality was analyzed for three different time frames. These time points were chosen because they yielded the best-fitting models, according to log-likelihood scores. ${ }^{26,27}$ Covariate adjustment in Cox models included age (introduced as an exponential term, which yielded a more robust association with mortality), gender, treatment variables and BP-monitoring variables. BMI was not included, as it did not predict mortality in this database. Confirmation of the time-dependent Cox calculations was obtained by logistic regression modeling examining the odds ratios for all-cause mortality at 2-10 years after the index ABPM session.

Data are expressed as mean \pm s.d. or as HR $(95 \% \mathrm{CI})$, unless specified otherwise. Two-sided nominal $P$-values $<0.05$ were considered significant. Calculations of the slope-related parameters and its inter-relationships were carried out using the statistical software, SYSTAT7.0, whereas calculations related to prognostic power were undertaken using SPSS 15.0 (both from SPSS Inc., Chicago, IL, USA).

\section{RESULTS}

\section{Study population}

Our ABPM service database included 3433 eligible individuals. Their demographic and clinical characteristics are depicted in Table 1. Mean age was $56 \pm 16$ years; $55 \%$ were women and $59 \%$ were treated with antihypertensive medications and $10 \%$ with hypoglycemic agents.

\section{Correlates of blood-pressure variability}

Median systolic and diastolic BP variabilities, defined as the coefficient of variation of 24-h ambulatory BP, were $10.9 \%$ (IQR 9.3-12.9) and $14.2 \%$ (IQR 12.3-16.7), respectively (Table 1 ). The mean ratio of systolic standard deviation to diastolic standard deviation (BPVR) was 1.33 (IQR 1.16-1.55). Various correlations were found between these log-transformed indices and clinical variables (Table 2).

Standard deviation of systolic and diastolic BP was linearly correlated with deciles of systolic and diastolic BP, respectively. Figure 1 shows that these linear associations are practically located on the same regression line, when BP variability is plotted against the corresponding BP decile $(r=0.984, P<0.0001)$. Importantly, the ratio of systolic to diastolic BP variability, BPVR, was found to be independent of mean arterial pressure (MAP); $r=0.013$ and $P=0.43$ (see Figure 1 inset for analysis by deciles and Table 2 for partial coefficients). This was true in both treated $(r=0.019)$ and untreated $(r=0.11)$ patients. In 
addition, Figure 2 shows that, although systolic and diastolic BP variabilities were found to be profoundly dependent on $R$ (the systolic $v s$. diastolic correlation coefficient), BPVR was nearly free of this association.

We reanalyzed 3401 patients (99\%) with separable BP reading in either wakefulness or during sleep (based on the patient's diary). BPVR calculated over 24-h correlated with both awake and sleep values ( $r=0.84$ and $r=0.65$, respectively). Awake and sleep BPVR displayed similar distributions, and were correlated $(r=0.38)$. BPVR value was slightly but significantly higher at wakefulness compared with that at sleep $(1.44 \pm 0.39$ vs. $1.35 \pm 0.43, P<0.001)$, suggesting

Table 1 Various demographic and ambulatory blood pressure monitoring characteristics of the study population according to treatment status ${ }^{a}$

\begin{tabular}{|c|c|c|c|}
\hline & untreated & treated & all patients \\
\hline number $(\%)$ & $1408(41)$ & $2025(59)$ & $3433(100)$ \\
\hline age, years & $47 \pm 16$ & $61 \pm 13^{\dagger}$ & $56 \pm 16$ \\
\hline gender, $\%$ women & 48 & 57 & 55 \\
\hline hypoglycemic treatment, \% & 3 & 13 & 10 \\
\hline body-mass-index, $\mathrm{kg} / \mathrm{m}^{2}$ & $26.5 \pm 4.4$ & $27.8 \pm 4.5^{\dagger}$ & $27.2 \pm 4.5$ \\
\hline follow-up, years & $8.3 \pm 4.0$ & $7.7 \pm 3.8^{\dagger}$ & $7.6 \pm 3.8$ \\
\hline clinic systolic BP, mm Hg & $142 \pm 19$ & $151 \pm 23^{\dagger}$ & $148 \pm 22$ \\
\hline clinic diastolic $\mathrm{BP}, \mathrm{mm} \mathrm{Hg}$ & $87 \pm 12$ & $84 \pm 13^{\dagger}$ & $85 \pm 13$ \\
\hline 24-hour systolic BP, $\mathrm{mm} \mathrm{Hg}$ & $135 \pm 15$ & $140 \pm 17^{\dagger}$ & $137 \pm 16$ \\
\hline 24-hour diastolic BP, $\mathrm{mm} \mathrm{Hg}$ & $80 \pm 10$ & $78 \pm 10^{\dagger}$ & $78 \pm 10$ \\
\hline systolic BP dip, \% & $10.8 \pm 7.1$ & $8.5 \pm 8.4^{\dagger}$ & $9.8 \pm 7.9$ \\
\hline diastolic BP dip, $\%$ & $14.8 \pm 8.4$ & $12.1 \pm 9.2^{\dagger}$ & $13.7 \pm 8.9$ \\
\hline 24-hour systolic CV, \% & $10.9(9.2-12.6)$ & $11.2(9.4-13.3)^{\dagger}$ & $10.9(9.3-12.9)$ \\
\hline 24-hour diastolic CV, \% & $14.4(12.5-16.7)$ & $14.2(12.1-16.8)$ & $14.2(12.3-16.7)$ \\
\hline $\operatorname{BPVR}\left(\right.$ s.d. $\left.._{(\mathrm{SBP})} / \mathrm{s} \cdot \mathrm{d}_{{ }_{(\mathrm{DBP})}}\right)$ & $1.25(1.10-1.45)$ & $1.40(1.21-1.62)^{\dagger}$ & $1.33(1.16-1.55)$ \\
\hline
\end{tabular}

$\mathrm{BP}$, blood pressure; BPVR, blood pressure variability ratio; $\mathrm{CV}$, coefficient of variation; $\mathrm{DBP}$, diastolic blood pressure; s.d., standard deviation; SBP, systolic blood pressure.

aData are presented as mean \pm s.d., median (IQR) or percentage, as appropriate.

$P<0.001$ by the appropriate statistical test.

Table 2 Partial correlation coefficients of blood pressure variability indices (log-transformed) and clinical characteristics in untreated $(n=1408)$ and treated $(n=2025)$ patients

\begin{tabular}{lrrrrrrr}
\hline \multirow{2}{*}{ Predictor } & \multicolumn{2}{c}{$\mathrm{CV}(\mathrm{SBP})$} & \multicolumn{2}{c}{$\mathrm{CV}(\mathrm{DBP})$} & \multicolumn{2}{c}{ BPVR } \\
& untreated & treated & untreated & treated & untreated & treated \\
\hline age, years & 0.068 & 0.110 & -0.160 & $0.071^{*}$ & 0.266 & $0.301^{*}$ \\
body-mass-index, $\mathrm{kg} / \mathrm{m}^{2}$ & 0.093 & $-0.053^{*}$ & 0.113 & 0.078 & 0.002 & $-0.112^{*}$ \\
mean arterial pressure, $\mathrm{mm} \mathrm{Hg}$ & -0.118 & -0.140 & -0.313 & $-0.197^{*}$ & 0.052 & 0.016 \\
mean arterial pressure dip, \% & 0.178 & 0.244 & 0.285 & 0.292 & -0.184 & -0.127 \\
systolic blood pressure, $\mathrm{mm} \mathrm{Hg}$ & 0.011 & -0.050 & -0.116 & -0.051 & 0.247 & 0.200 \\
diastolic blood pressure, $\mathrm{mm} \mathrm{Hg}$ & -0.189 & -0.189 & -0.403 & -0.283 & -0.092 & -0.144 \\
pulse pressure, mm Hg & 0.192 & $0.075^{*}$ & 0.228 & $0.143^{*}$ & 0.409 & $0.355^{*}$ \\
\hline
\end{tabular}

BPVR, blood pressure variability ratio; $\mathrm{CV}$, coefficient of variation; $\mathrm{DBP}$, diastolic blood pressure; SBP, systolic blood pressure.

Partial correlation values $(r)$ were derived by multivariable linear regression models, which

included age, gender, body mass index, treatment for diabetes and MAP dip.

${ }^{*} P$-value $<0.05$ for comparison between untreated and treated patients. that, basically, BPVR calculated over $24-\mathrm{h}(1.39 \pm 0.33)$ fairly represents both day and night values and relates to a property that is insensitive to the circadian cycle.

Comparisons between BPVR and the ambulatory arterial stiffness index determined by different methods

BPVR and Slope(bisect) are highly correlated $(r=0.87, P<0.001)$. Figure 3 shows that the magnitude of this association depends on the correlation $R$ between systolic and diastolic BP for individual patients, reaching $r=0.996(P<0.0001)$ for the vast majority of patients $(n=3086,90 \%)$ in whom $R>0.5$, with $r=0.439$ for the rest $(R \leqslant 0.5)$. Very similar correlations are found for the corresponding AASI(BPVR) and AASI(bisect) (see Methods). The correlation between AASI(BPVR) and AASI(standard) is $r=0.830$ for $R>0.5$, with $r=0.480$ for $R \leqslant 0.5$, which may reflect the explicit dependence of AASI(standard) on $R$ (see Appendix).

\section{Blood pressure variability and all-cause mortality}

During the mean follow-up of 7.6 years, 237 patients died. After adjustment for demographic and treatment variables, as well as for MAP and MAP dip, the variability (CV) of systolic BP was marginally associated with all-cause mortality; hazards ratio (HR) 1.12 (95\% confidence intervals $(95 \% \mathrm{CI}) 0.97-1.28, P=0.1)$ per 1 s.d. increase. In a parallel model of diastolic BP variability, HR was 1.04 (0.92-1.16) per 1 s.d.. However, an analogous model of the BP variability ratio, BPVR, yielded a significant HR of $1.21(1.05-1.40)$ per 1 s.d. increase in log-transformed BPVR $(P=0.007)$. Introduction of BPVR did not materially change the prognostic significance of other established parameters, such as age, systolic BP, BP dipping, and so on ${ }^{28}$ (data not shown). In the latter model, however, the proportional hazards assumption was not valid (see Methods). More specifically, the hazards ratio per unit increase in BPVR seemed to dampen with time (see also Supplementary mortality figures). Thus, time-dependent covariates were introduced. We chose covariates that partitioned follow-up time at 1.5 and 7 years, as this yielded the best-fitting model. ${ }^{26,27}$ With these terms in the model, hazards ratios per 1 s.d. increase in log-transformed BPVR were 2.21 (95\% CI 1.36-3.59) in the first 1.5 years, 1.28 (1.06-1.54) between 1.5 and 7 years and $0.90(0.68-1.20)$ thereafter. A simplified analysis of mortality by quartiles of BPVR, displayed in Figure 4, shows that compared with the first quartile, patients at the

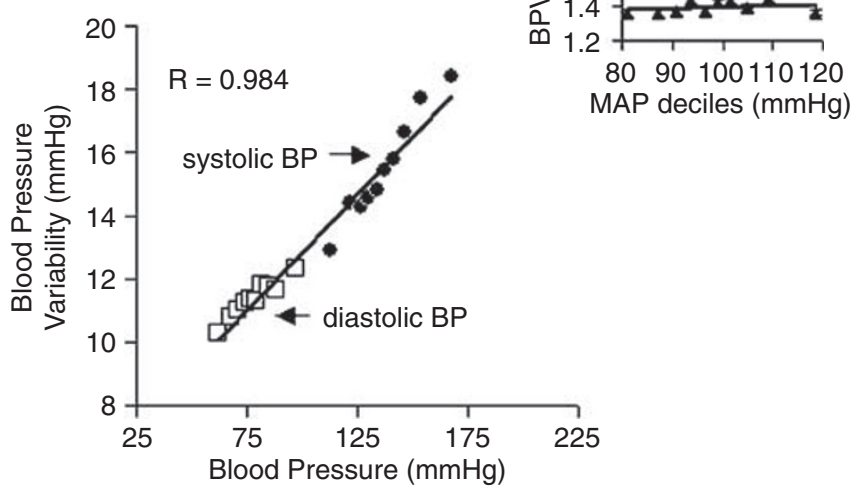

Figure 1 A scatter plot illustrating the standard deviation of blood pressure vs. 24-h blood pressure (partitioned by deciles). Diastolic blood pressure (open squares) and systolic blood pressure (black circles) are plotted separately. Correlation was tight; $R=0.984, P<0.0001$. Inset: the blood pressure variability ratio (BPVR) plotted vs. mean arterial blood pressure deciles, showing no apparent association: $r=0.029, P=0.13$. 

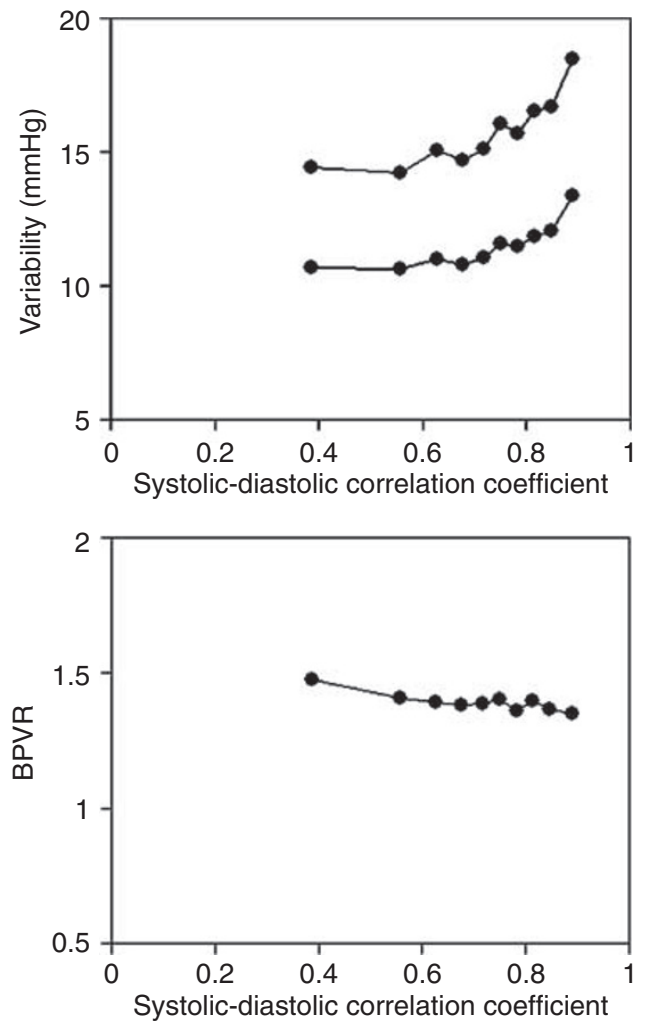

Figure 2 Systolic and diastolic blood pressure (BP) variability (top) or blood pressure variability ratio (BPVR) (bottom) are plotted against deciles of the correlation coefficient of the linear relationship between systolic and diastolic BP $(R)$. Deciles of $R$ are adjusted for age, gender, body mass index (BMI) and systolic blood pressure (SBP) or diastolic blood pressure (DBP) (top) or mean arterial pressure (MAP) (bottom), by means of analysis of covariance (ANCOVA) $(F=29.2 / 43.8$ for SBP/DBP, $P<0.0001$; $F=2.36$ for BPVR, $P=0.012$ ). Standard error is within the size of the data points. highest quartile of BPVR had increased mortality up to 7 years, HR $2.30(1.26-4.21)$

We further explored the prognostic ability of BPVR compared with that of standard and symmetric AASI with additional adjustment for 24-h pulse pressure (PP) (Table 3 and Supplementary mortality figures). All-cause mortality during the first 1.5 years of follow-up, adjusted for demographic as well as for monitoring parameters (including 24-h MAP, 24-h PP and dipping magnitude), increased with BPVR: HR 1.97 (1.20-3.21) per 1 s.d.; AASI(standard): HR 1.58 (0.95-2.64) and AASI(bisect): HR 1.35 (1.05-1.75). Between 1.5 and 7 years, the corresponding hazards ratios had borderline significance: BPVR, 1.17 (0.96-1.41); AASI(standard), 1.19 (0.94-1.51); and AASI (bisect); 1.10 (0.92-1.32). Thereafter, these indices were no longer associated with increased mortality (Table 3 and Supplementary mortality figures). In sensitivity analyses limited to individuals treated or not treated for hypertension, Cox coefficients did not differ from those derived for the total population (data not shown).

We used logistic regression to confirm the results of time-dependent Cox proportional hazards models. We examined the odds ratios for all-cause mortality at 2 years $(n=2796), 5$ years $(n=2187)$ and 7 years $(n=1542)$ after the index ambulatory monitoring session (Table 4). The results were consistent with the Cox models in showing a declining strength of association with increasing duration of follow-up.

\section{DISCUSSION}

Our foremost finding that BPVR - the ratio of the standard deviation of systolic ambulatory BP to that of diastolic ambulatory BP-is nearly identical to the slope of the systolic $v$ s. diastolic BP relationship estimated by the bisector regression method should not come as a surprise, as it is the realization of a mathematical relationship (the same slope calculated by two symmetric regression methods, see Appendix). Nevertheless, the identity (which reaches $r=0.996$ in patients with linearly related s.d.(SBP) and s.d.(DBP) between these variables is impressive. The main importance of this relationship is

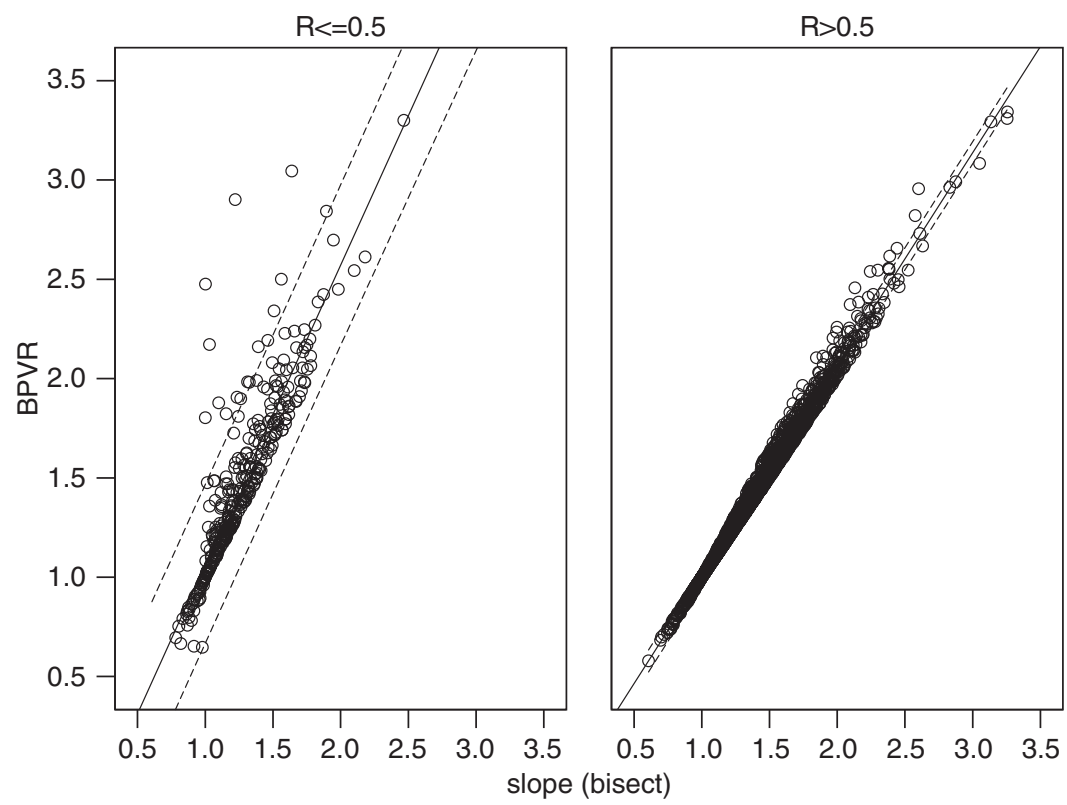

Figure 3 Scatter plots illustrating the regression line (and $95 \% \mathrm{Cl}$ ) between blood pressure variability ratio (BPVR) and Slope(bisect), for patients with linearly $(R>0.5)$ related systolic and diastolic 24-h blood pressure (BP) (right plot, correlation coefficient $r=0.996)$ and for patients with low $(R \leqslant 0.5)$ correlation between systolic and diastolic BP (left plot, $r=0.439$ ). 


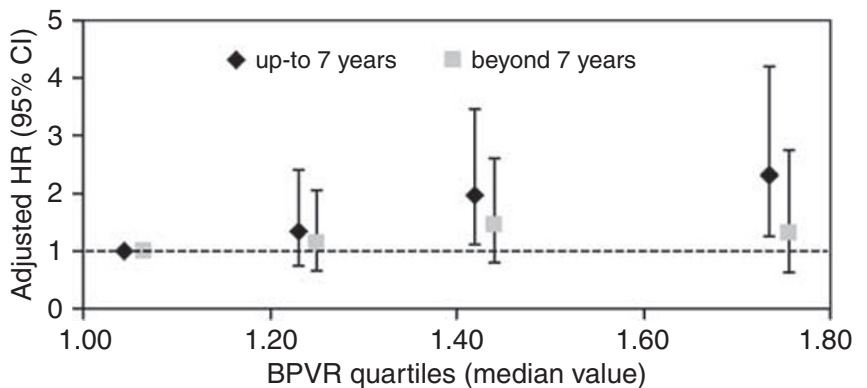

Figure 4 All-cause mortality by quartiles of blood pressure variability ratio (BPVR). Hazards ratios and 95\% confidence intervals (bars), plotted against the median BPVR value of each quartile, were generated by a Cox proportional model with a single time-dependent partition at 7 years. Covariates included in the model: age (exponential term), gender, treatment for hypertension and diabetes, mean arterial pressure and mean arterial blood pressure dip. $P$-value for the trend up to 7 years (black diamonds) $=0.02 ; P$-value for the trend beyond 7 years (gray squares) $=0.6$.

Table 3 Association of BPVR and other Slope-related parameters with all-cause mortality: Cox-proportional hazards models

\begin{tabular}{lccc}
\hline \multirow{2}{*}{ Predictor } & \multicolumn{3}{c}{ Adjusted hazards-ratios for all-cause mortality per 1 s.d. } \\
& $0-18 \mathrm{mo}$. & $18-84 \mathrm{mo}$. & $>84$ mo. (7 years) \\
\hline AASI(standard) & $1.58(0.95-2.64)$ & $1.19(0.94-1.51)$ & $0.86(0.62-1.20)$ \\
AASI(bisect) & $1.35(1.05-1.75)$ & $1.10(0.92-1.32)$ & $0.88(0.66-1.18)$ \\
AASI(BPVR) & $2.38(1.20-4.73)$ & $1.20(0.96-1.49)$ & $0.89(0.69-1.15)$ \\
Slope(bisect) & $1.76(1.24-2.50)$ & $1.15(0.97-1.36)$ & $0.89(0.69-1.16)$ \\
BPVR & $1.97(1.20-3.21)$ & $1.17(0.96-1.41)$ & $0.84(0.64-1.12)$ \\
\hline
\end{tabular}

AASI, ambulatory arterial stiffness index; BPVR, blood pressure variability ratio; mo., months. aLog-transformed values were used for BPVR.

Mortality is expressed as hazards ratios (and 95\% confidence intervals) per 1 standard deviation of the index predictor. Hazards ratios were derived from Cox proportional models with timedependent covariates yielding partitioned results as displayed (see Methods and supplementary figures). Covariates in the model included age (exponential term), sex, treatment for diabetes and hypertension, 24-h mean arterial pressure and pulse pressure, and mean arterial pressure dip.

Table 4 Association of BPVR and other Slope-related parameters with all-cause mortality: logistic regression models

\begin{tabular}{lccc}
\hline Predictor & \multicolumn{2}{c}{ Adjusted odds-ratios for all-cause mortality per 1 s.d. } \\
& 2-years & 5 -years & 7 -years \\
\hline AASI(standard) & $1.59(1.02-2.47)$ & $1.32(1.00-1.74)$ & $1.19(0.90-1.57)$ \\
AASI(bisect) & $1.21(0.91-1.61)$ & $1.16(0.96-1.41)$ & $1.20(0.98-1.46)$ \\
AASI(BPVR) & $1.64(1.01-2.66)$ & $1.35(1.03-1.76)$ & $1.28(1.00-1.64)$ \\
Slope(bisect) & $1.34(0.97-1.84)$ & $1.25(1.02-1.53)$ & $1.26(1.04-1.54)$ \\
BPVR & $1.52(1.03-2.26)$ & $1.31(1.04-1.65)$ & $1.25(1.01-1.56)$ \\
\hline
\end{tabular}

AASI, ambulatory arterial stiffness index; BPVR, blood pressure variability ratio.

aLog-transformed values were used for BPVR.

Odds ratios (and 95\% confidence intervals) for all-cause mortality per 1 standard deviation of the index predictor were derived with logistic regression models. Covariates in the models included age (exponential term), sex, treatment for diabetes and hypertension, 24-h mean arterial pressure and pulse pressure, and mean arterial pressure dip. At 2-years, $n=2796$; 5 -years, $n=2187 ; 7$-years, $n=1542$. At 10 years $(n=933)$ odds ratios were insignificant for all predictors (not shown). that it eliminates the need for all raw data points and statistical software to regress them, as the s.d. of the 24-h ambulatory BP is available from any report.

AASI and its related slope have been shown to predict outcome, ${ }^{5,7,8,29}$ as well as target organ damage. ${ }^{6,30}$ However, the importance of these parameters as measures of arterial properties has been questioned by downplaying the role of AASI as a genuine correlate of arterial stiffness ${ }^{31,32}$ because of its strong dependence on age, gender, ${ }^{31,32}$ pulse pressure ${ }^{32}$ and nocturnal BP decline. ${ }^{3,25,32}$ We report here that the slope-related indices, AASI, AASI(bisect), Slope(bisect) and BPVR, all predict mortality to a certain extent, and that the indices derived from the proper symmetric regression ${ }^{8}$ and the calculated BPVR, tend to predict even better. Indeed, after adjustment for age, ambulatory BP level, 24-h PP (an arterial stiffness characteristic) and the corresponding decline of ambulatory BP or PP during sleep (an independent mortality predictor in this patient population ${ }^{28}$ ), BPVR remains significantly associated with increased mortality.

The slope of systolic vs. diastolic BP is unlikely to link directly to arterial stiffness, as the latter is a strictly BP-related variable, whereas BPVR (and related indices) are independent of mean arterial pressure. $^{3}$ Theoretical considerations predicting that the systolic $v s$. diastolic slope is equivalent to the relative increase of arterial stiffness during systole ${ }^{2}$ are supported by its being typically greater than unity, a reflection of a higher systolic than diastolic stiffness. ${ }^{3}$ Folkow et al. ${ }^{33,34}$ recognized decades ago that the arterial reactivity to pressors is higher in hypertensive than in normotensive rats. Such increased vascular reactivity, also found in human hypertension, ${ }^{35}$ is believed to reflect a higher arterial cross-sectional area and subsequently smaller lumen when the vessel wall is hypertrophied. Indeed, such structural arterial changes underlie an increased risk in hypertension. ${ }^{36}$ Thus, the relationship between arterial structural and functional properties, and $\mathrm{BP}$ reactivity to different stressors, which defines BP variability, rest on sound physiological grounds. In other words, a greater variation of ambulatory BP during systole than during diastole may be an integrated reflection of arterial structural and functional properties that can be captured by ambulatory BP-derived variables such as the s.d. of BP.

An important observation is that, although the s.d. of both systolic and diastolic BP is tightly related to $R$ (the correlation coefficient of the systolic vs. diastolic BP), the ratio of systolic-to-diastolic BP variability (BPVR) is much less dependent on $R$ and hence less influenced by outliers (see Figure 2).

The waning of the prediction of mortality with time may seem as a weakness of BPVR and AASI (the validity of the Cox proportional hazards assumption was not explicitly reported in some of the earlier AASI outcome studies, for example, Dolan et al..$^{5}$ and Kikuya et al. ${ }^{29}$ ). Prediction was strong up to 18 months, fair up to 7 years and nonsignificant thereafter. However, during these periods of time, many aspects may change, including BP control and the use of medications that may affect arterial structure. Changes in structure would affect BP reactivity, and hence its variability, during the varying stresses of the awake and asleep periods. Indeed, in the largest hypertension study to date, ${ }^{26}$ the effect of antihypertensive medications on congestive heart failure outcome was also blunted with time. As the relationship of BP to heart failure is indisputable, similarly the blunting over time of the BPVR prediction of all-cause mortality should not be an argument to nullify its importance. This limitation of our study is related to the known regression dilution bias. ${ }^{37}$ Introduction of follow-up measurements, had they been available, may have altered this trend. Obviously, a better understanding of the underlying physiology represented by BPVR will enhance the understanding of this phenomenon. 
Our study has several other limitations. It reflects a referred population and may not represent the hypertensive or general population. More than $50 \%$ of the patients were treated for hypertension, and antihypertensive therapy is known to reduce BP variability. ${ }^{17,38}$ We used the 24-h s.d. of BP, which incorporates in it the circadian variation, and BPVR might be improved by appropriate weighing of awake and asleep measurements. ${ }^{39}$ However, as we consider that the 24-h association between systolic and diastolic BP yields a reliable property that incorporates the extremes of circadian BP changes, we used the simple s.d. This may underestimate the predictive power of BPVR. Baseline cardiovascular comorbidities and risk factors were largely unavailable for outcome analyses. Their absence does not allow an accurate estimation of the prognostic information depicted in BPVR. However, a comparison of BPVR with AASI and blood pressure variability is still valid.

In conclusion, although the physiological meaning of the slope relating systolic to diastolic ambulatory BP is not well understood, the potential of BPVR and the other slope-related parameters to reveal vascular properties deserves exploration. Indeed, some readily available slope-related indices, including the BPVR, which may be viewed also as a derivation of BP variability, should be further evaluated as practical measures of vascular health. ${ }^{40}$ We should consider such predictors at a time when individual global risk assessment is becoming incorporated in hypertension guidelines. ${ }^{41}$

1 O'Brien E. Ambulatory blood pressure measurement: a trove of hidden gems? Hypertension 2006; 48: 364-365.

2 Gavish B. Repeated blood pressure measurements may probe directly an arterial property. Am J Hypertens 2000; 13: 190A (abstract).

3 Gavish B, Ben Dov IZ, Bursztyn M. Linear relationship between systolic and diastolic blood pressure monitored over 24-h: assessment and correlates. J Hypertens 2008; 26: 199-209.

4 Li Y, Wang JG, Dolan E, Gao PJ, Guo HF, Nawrot T, Stanton AV, Zhu DL, O'Brien E, Staessen JA. Ambulatory arterial stiffness index derived from 24-h ambulatory blood pressure monitoring. Hypertension 2006; 47: 359-364.

5 Dolan E, Thijs L, Li Y, Atkins N, McCormack P, McClory S, O'Brien E, Staessen JA, Stanton AV. Ambulatory arterial stiffness index as a predictor of cardiovascular mortality in the Dublin Outcome Study. Hypertension 2006; 47 365-370.

6 Leoncini G, Ratto E, Viazzi F, Vaccaro V, Parodi A, Falqui V, Conti N, Tomolillo C, Deferrari G, Pontremoli R. Increased Ambulatory Arterial Stiffness Index is associated with target organ damage in primary hypertension. Hypertension 2006; 48: 397-403.

7 Hansen TW, Staessen JA, Torp-Pedersen C, Rasmussen S, Li Y, Dolan E, Thijs L, Wang JG, O'Brien E, Ibsen H, Jeppesen J. Ambulatory arterial stiffness index predicts stroke in a general population. J Hypertens 2006; 24: 2247-2253.

8 Ben Dov IZ, Gavish B, Kark JD, Mekler J, Bursztyn M. A modified ambulatory arterial stiffness index is independently associated with all-cause mortality. J Hum Hypertens 2008; 22: 761-766.

9 Kermack KA, Haldane JB. Organic correlation and allometry. Biometrika 1950; 37 30-41.

10 von Eye A, Schuster C. Regression Analysis for Social Science. Academic Press: San Diego, CA, 1998, 209-236.

11 Mancia G, Ferrari A, Gregorini L, Parati G, Pomidossi G, Bertinieri G, Grassi G, di Rienzo M, Pedotti A, Zanchetti A. Blood pressure and heart rate variabilities in normotensive and hypertensive human beings. Circ Res 1983; 53: 96-104.

12 Parati G, Pomidossi G, Albini F, Malaspina D, Mancia G. Relationship of 24-h blood pressure mean and variability to severity of target-organ damage in hypertension. J Hypertens 1987; 5: 93-98.

13 Tatasciore A, Renda G, Zimarino M, Soccio M, Bilo G, Parati G, Schillaci G, De Caterina R. Awake systolic blood pressure variability correlates with target-organ damage in hypertensive subjects. Hypertension 2007; 50: 325-332.

14 Shintani Y, Kikuya M, Hara A, Ohkubo T, Metoki H, Asayama K, Inoue R, Obara T, Aono Y, Hashimoto T, Hashimoto J, Totsune K, Hoshi H, Satoh H, Imai Y. Ambulatory blood pressure, blood pressure variability and the prevalence of carotid artery alteration: the Ohasama study. J Hypertens 2007; 25: 1704-1710.

15 Sander D, Kukla C, Klingelhofer J, Winbeck K, Conrad B. Relationship between circadian blood pressure patterns and progression of early carotid atherosclerosis: a 3-year follow-up study. Circulation 2000; 102: 1536-1541.

16 Pringle E, Phillips C, Thijs L, Davidson C, Staessen JA, de Leeuw PW, Jaaskivi M, Nachev C, Parati G, O'Brien ET, Tuomilehto J, Webster J, Bulpitt CJ, Fagard RH. Systolic blood pressure variability as a risk factor for stroke and cardiovascular mortality in the elderly hypertensive population. J Hypertens 2003; 21: 2251-2257.

17 Kikuya M, Hozawa A, Ohokubo T, Tsuji I, Michimata M, Matsubara M, Ota M, Nagai K, Araki T, Satoh H, Ito S, Hisamichi S, Imai Y. Prognostic significance of blood pressure and heart rate variabilities: The Ohasama Study. Hypertension 2000; 36: 901-906.

18 Grin JM, McCabe EJ, White WB. Management of hypertension after ambulatory blood pressure monitoring. Ann Intern Med 1993; 118: 833-837.

19 Groppelli A, Omboni S, Parati G, Mancia G. Evaluation of noninvasive blood pressure monitoring devices Spacelabs 90202 and 90207 versus resting and ambulatory 24-hour intra-arterial blood pressure. Hypertension 1992; 20: 227-232.

20 White WB, Lund-Johansen P, McCabe EJ, Omvik P. Clinical evaluation of the Accutracker II ambulatory blood pressure monitor: assessment of performance in two countries and comparison with sphygmomanometry and intra-arterial blood pressure at rest and during exercise. J Hypertens 1989; 7: 967-975.

21 White WB, Lund-Johansen P, Omvik P. Assessment of four ambulatory blood pressure monitors and measurements by clinicians versus intraarterial blood pressure at rest and during exercise. Am J Cardiol 1990; 65: 60-66.

22 Perk G, Mekler J, Bursztyn M. Ambulatory pulse pressure is a relatively sleepindependent variable. J Hypertens 2003; 21: 723-728.

23 Owens P, Atkins N, O'Brien E. Diagnosis of white coat hypertension by ambulatory blood pressure monitoring. Hypertension 1999; 34: 267-272.

24 Dechering DG, Adiyaman A, van der SM, Thien T. Interstudy variability in the ambulatory arterial stiffness index. Hypertension 2007; 50: e65.

25 Schillaci G, Parati G, Pirro M, Pucci G, Mannarino MR, Sperandini L, Mannarino E. Ambulatory arterial stiffness index is not a specific marker of reduced arterial compliance. Hypertension 2007; 49: 986-991.

26 Davis BR, Piller LB, Cutler JA, Furberg C, Dunn K, Franklin S, Goff D, Leenen F, Mohiuddin S, Papademetriou V, Proschan M, Ellsworth A, Golden J, Colon P, Crow R. Role of diuretics in the prevention of heart failure: the Antihypertensive and Lipid-Lowering Treatment to Prevent Heart Attack Trial. Circulation 2006; 113: 2201-2210.

27 Klein JP, Moeschberger ML. Survival Analysis: Techniques for Censored and Truncated Regression. Springer-Verlag: New York, NY, 1997.

28 Ben Dov IZ, Kark JD, Ben Ishay D, Mekler J, Ben Arie L, Bursztyn M. Predictors of allcause mortality in clinical ambulatory monitoring: unique aspects of blood pressure during sleep. Hypertension 2007; 49: 1235-1241.

29 Kikuya M, Staessen JA, Ohkubo T, Thijs L, Metoki H, Asayama K, Obara T, Inoue R, Li Y, Dolan E, Hoshi H, Hashimoto J, Totsune K, Satoh H, Wang JG, O'Brien E, Imai Y. Ambulatory arterial stiffness index and 24-hour ambulatory pulse pressure as predictors of mortality in Ohasama, Japan. Stroke 2007; 38: 1161-1166.

30 Mule G, Cottone S, Cusimano P, Incalcaterra F, Giandalia M, Costanzo M, Nardi E, Palermo A, Geraci C, Costa R, Cerasola G. Inverse relationship between ambulatory arterial stiffness index and glomerular filtration rate in arterial hypertension. $A m \mathrm{~J}$ Hypertens 2008; 21: 35-40.

31 Jerrard-Dunne P, Mahmud A, Feely J. Ambulatory arterial stiffness index, pulse wave velocity and augmentation index-interchangeable or mutually exclusive measures? J Hypertens 2008; 26: 529-534.

32 Baumann M, Dan L, Nurnberger J, Heemann U, Witzke O. Association of ambulatory arterial stiffness index and brachial pulse pressure is restricted to dippers. $J$ Hypertens 2008; 26: 210-214.

33 Folkow B, Hallback M, Lundgren Y, Weiss L. Background of increased flow resistance and vascular reactivity in spontaneously hypertensive rats. Acta Physiol Scand 1970; 80: 93-106.

34 Folkow B, Hallback M, Lundgren Y, Weiss L. Structurally based increase of flow resistance in spontaneously hypertensive rats. Acta Physiol Scand 1970; 79: 373-378.

35 Doyle AE, Fraser JR. Vascular reactivity in hypertension. Circ Res 1961; 9: 755-761.

36 Rizzoni D, Porteri E, Boari GE, De Ciuceis C, Sleiman I, Muiesan ML, Castellano M, Miclini M, Agabiti-Rosei E. Prognostic significance of small-artery structure in hypertension. Circulation 2003; 108: 2230-2235.

37 Clarke R, Shipley M, Lewington S, Youngman L, Collins R, Marmot M, Peto R. Underestimation of risk associations due to regression dilution in long-term follow-up of prospective studies. Am J Epidemiol 1999; 150: 341-353.

38 Mancia G, Bombelli M, Facchetti R, Madotto F, Corrao G, Trevano FQ, Grassi G, Sega R. Long-term prognostic value of blood pressure variability in the general population: results of the Pressioni Arteriose Monitorate e Loro Associazioni Study. Hypertension 2007; 49: 1265-1270.

39 Bilo G, Giglio A, Styczkiewicz K, Caldara G, Maronati A, Kawecka-Jaszcz K, Mancia G, Parati G. A new method for assessing 24-h blood pressure variability after excluding the contribution of nocturnal blood pressure fall. J Hypertens 2007; 25: 2058-2066

40 Lakatta EG. Arterial and cardiac aging: major shareholders in cardiovascular disease enterprises: Part III: cellular and molecular clues to heart and arterial aging. Circulation 2003; 107: 490-497.

41 Mancia G, De Backer G, Dominiczak A, Cifkova R, Fagard R, Germano G, Grassi G, Heagerty AM, Kjeldsen SE, Laurent S, Narkiewicz K, Ruilope L, Rynkiewicz A, Schmieder RE, Boudier HA, Zanchetti A, Vahanian A, Camm J, De Caterina R, Dean V, Dickstein K, Filippatos G, Funck-Brentano C, Hellemans I, Kristensen SD, McGregor K, Sechtem U, Silber S, Tendera M, Widimsky P, Zamorano JL, Erdine S, Kiowski W, Agabiti-Rosei E, Ambrosioni E, Lindholm LH, Viigimaa M, Adamopoulos S, AgabitiRosei E, Ambrosioni E, Bertomeu V, Clement D, Erdine S, Farsang C, Gaita D, Lip G, Mallion JM, Manolis AJ, Nilsson PM, O'Brien E, Ponikowski P, Redon J, Ruschitzka F, 
Tamargo J, van Zwieten P, Waeber B, Williams B. 2007 Guidelines for the management of arterial hypertension: The Task Force for the Management of Arterial Hypertension of the European Society of Hypertension (ESH) and of the European Society of Cardiology (ESC). J Hypertens 2007; 25: 1105-1187.

\section{APPENDIX}

The relationship between slope-related parameters calculated by different regression methods

Figure A1 summarizes the different slopes determined for the same data: ${ }^{10}$ the 'direct (standard) regression' line \#1 (slope B1) minimizes the sum of the square of the deviations of the individual data points in the systolic blood pressure (SBP) direction only; line \#2 (slope B2) obtained by 'reversed regression' minimizes the same sum, but for the diastolic blood pressure (DBP) direction only; the bisector of the angle between the previous lines-line \#3 (slope Bbisect)—establishes a symmetric procedure; the 'reduced major axis' regression line \#4 (slope B) minimizes the total area of the triangles formed by the individual data points when connected to line \#4 by passing vertical and horizontal lines through each point. This method is symmetric by definition. All regression lines pass through the average blood pressure (BP) point and correspond to the same correlation coefficient $R$. It is important to mention that the relationships between the parameters are purely mathematical and do not represent 'experimental error'. As shown here, all parameters can be expressed by three measurable quantities: the standard deviations of SBP and DBP and the correlation coefficient $R$ of both variables.

The following is a compact summary of the statistical background and the derivation of specific expressions relevant to this study: Let $x$

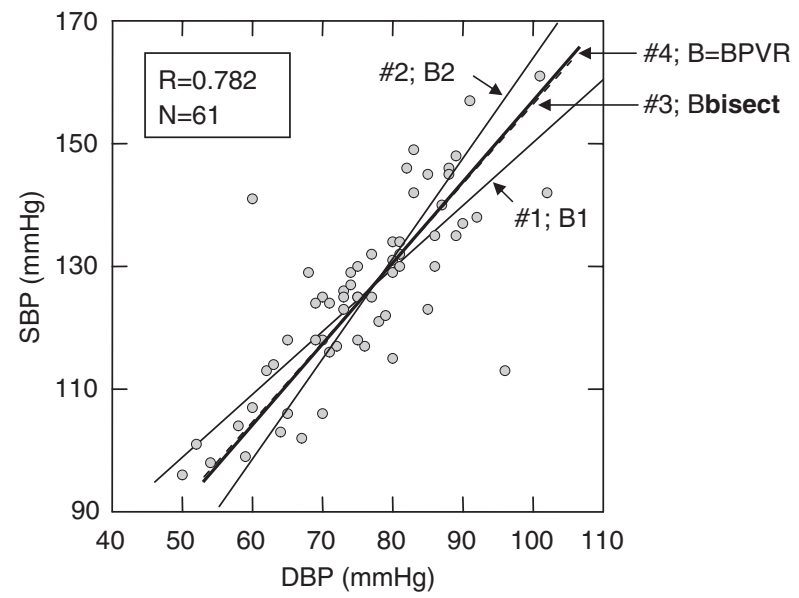

Figure $\mathbf{A} 1 \mathrm{~A}$ case report showing the best-fitted lines obtained by different regression methods and the corresponding slopes: slope B1 of line \#1 was obtained by 'direct (standard) regression' (systolic blood pressure (SBP)-vs. diastolic blood pressure (DBP) data plot); slope B2 of line \#2 by 'reversed regression', that is, standard regression applied to DBP-vs.-SBP data plot and taking the reciprocal value of the slope when plotted in the SBP-versusDBP frame; slope Bbisect of line \#3 (dashed) was obtained by symmetric regression of the bisector type (line \#3 is the bisector of the acute angle between lines \#1 and \#2); and the slope blood pressure variability ratio (BPVR) of line \#4 represents the ratio between the SBP variation over the DBP variation (BPVR), as obtained by the symmetric regression of the 'reduced major axis' type. ${ }^{22}$ BPVR also equals the geometrical mean of the slopes of lines \#1 and \#2, that is, to (B1B2) ${ }^{1 / 2}$. All details are given in Appendix. Note that the correlation coefficient is independent of the regression method used. and $y$ stand for diastolic blood pressure (DBP) and systolic blood pressure (SBP), respectively. Let $\Delta x$ and $\Delta y$ stand for the deviations of the individual data points from the mean $x$ and $y$ values, respectively. Let Sxx, Syy and Sxy be, respectively, the average value of $\Delta x^{2}, \Delta y^{2}$ and $\Delta x \times \Delta y$ (Sxy equals Syx). Let $s_{x}$ and $s_{y}$ be the standard deviations corresponding to $x$ and $y$ data, respectively; then Sxx equals $s_{x}^{2}$ and Syy equals $s_{y}{ }^{2}$. The correlation coefficient $R$ is defined by $\operatorname{Sxy} /(\operatorname{SxxSyy})^{1 / 2}$. Using these definitions, the standard expression for B1 is Sxy/Sxx. ${ }^{22}$ By switching the axes, that is, $x \rightarrow y$ and $y \rightarrow x$, we obtain the slope B2', which equals Sxy/Syy (Sxy equals Syx by definition). However, when viewed in the original axes $(y v s . x)$, it corresponds to slope B2, which

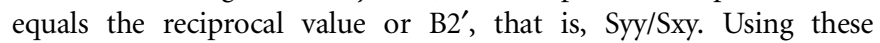
definitions, it is easy to show that

$$
s_{y} / s_{x}=\mathrm{B} 1 / R=R \mathrm{~B} 2
$$

It can be shown ${ }^{9,10}$ that the slope $B$ obtained by the 'reduced major axis' regression procedure equals $(\mathrm{B} 1 \mathrm{~B} 2)^{1 / 2}$. Using equation (A.1) and assuming that the regression line has a positive slope, we find the following:

$$
B=s_{y} / s_{x} \equiv \mathrm{BPVR}
$$

For $R$ smaller than 1 , equation (A.1) shows that $B$ is always greater than B1 and smaller than B2, as shown in Figure A1. For $R$ equal to 1 , all these lines coincide.

Using the standard definition of AASI by 1-[slope of the regression line obtained from DBP vs. SBP plot] or, in our notation, $1-\mathrm{B}^{\prime}$, which equals $1-1 / \mathrm{B} 2$, we obtain

$$
\text { AASI (standard) }=1-R / B
$$

The R-dependence of AASI(standard) has been shown to provide artifactual outcomes. ${ }^{3}$ In this paper, we would re-define this parameter in a symmetric way as follows:

$$
\text { AASI }(\mathrm{BPVR})=1-1 / B
$$

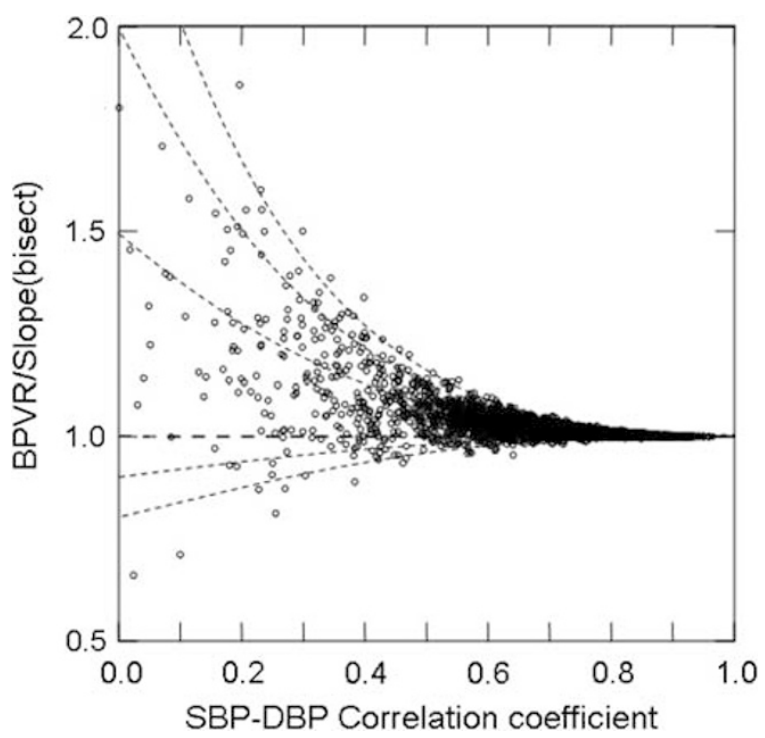

Figure A2 The ratio between the systolic blood pressure (SBP)-on-diastolic blood pressure (DBP) slope estimated by blood pressure variability ratio (BPVR) and that obtained using the bisector method (Slope(bisect)) ${ }^{3}$. The dashed lines are the theoretical curves given by equation (A.8) for the BPVR values $2.5,2.0,1.5,1.0,0.9$ and 0.8 (top to bottom). The plot shows that for the $\sim 90 \%$ data for which the SBP-DBP correlation coefficient is greater than 0.5 , both slope estimations are rather close. 
Using the bisector regression method the slope is given as follows: ${ }^{10}$

Bbisect $=\left\{\mathrm{B} 1 * \mathrm{~B} 2-1+\left[\left(1+\mathrm{B} 1^{2}\right)\left(1+\mathrm{B}^{2}\right)\right]^{1 / 2}\right\} /(\mathrm{B} 1+\mathrm{B} 2)$

As B1 and B2 are equal, according to equations (A.1) and (A.2), to $B R$ and $B / R$, equation (A.5) can be rewritten as follows:

$$
\text { Bbisect }=\left\{B^{2}-1+\left[\left(1+B^{2} R^{2}\right)\left(1+B^{2} / R^{2}\right)\right]^{1 / 2}\right\} /[B(R+1 / R)]
$$

Using this definition, we have already defined a symmetric AASI in the following form: ${ }^{3}$

$$
\text { AASI (bisect) }=1-1 / \text { Bbisect }
$$

The similarity between BPVR and Bbisect can be evaluated by the range at which $\mathrm{B} / \mathrm{Bbisect}$ is close to 1 using the expression

$$
\mathrm{B} / \text { Bbisect }=\mathrm{B}^{2}(R+1 / R) /\left\{\mathrm{B}^{2}-1+\left[\left(1+R^{2} \mathrm{~B}^{2}\right)\left(1+\mathrm{B}^{2} / R^{2}\right)\right]^{1 / 2}\right\}
$$

For $R \rightarrow 1$, equation (A.8) yields 1, suggesting that for high enough $R$ values, $B$ and Bbisect display close values. On the other extreme, for $R$ much lesser than 1 (taking into account that $B$ is typically lesser than 3 ), equation (A.8) is simplified into $B$, suggesting that in this range, larger deviations from 1 are associated with greater $B$ values. Figure A2 shows the superposition of equation (A.8) on the actual data.

Supplementary Information accompanies the paper on Hypertension Research website (http://www.nature.com/hr) 